\title{
Assessment of workload: intrauterine device/intrauterine system provision
}

\author{
Varghese A George, Meera Kishen
}

\begin{abstract}
The Faculty of Family Planning and Reproductive Health Care (FFPRHC) has recently published Service Standards for Workload in Contraception, which state that a minimum of 20 minutes should be made available for intrauterine device/hormonal system (IUD/IUS) provision. This document acknowledges that there is currently little formal evidence relating to assessment of time taken for providing various contraceptive methods. The Abacus Clinics in Liverpool, UK provide an average of 1300 IUD/IUS fittings in a year. We monitored the time taken for IUD/IUS provision over a 4-week period. Our study revealed that the average time taken for all types of IUD/IUS provision is significantly more than the minimum recommended by the FFPRHC.
\end{abstract}

J Fam Plann Reprod Health Care 2006; 32(3): 171-172 (Accepted 4 April 2006)

\section{Key message points \\ - Intrauterine device/intrauterine system (IUD/IUS) provision in accordance with Good Medical Practice and Faculty Standards requires more time than is currently recognised. \\ - Training activity significantly increases the consultation time for IUD/IUS provision. \\ - The value of routine prior counselling visits in reducing the time needed for IUD/IUS fitting is debatable.}

\section{Background}

The Faculty of Family Planning and Reproductive Health Care (FFPRHC) has recently published Service Standards for Workload in Contraception ${ }^{1}$ for use in family planning clinics. This document recommends that a minimum of 20 minutes should be made available for intrauterine device/intrauterine system (IUD/IUS) insertion by a doctor and nurse team.

Abacus Clinics in Liverpool, UK provide around 1300 IUD/IUS fittings per year. Nearly half of the IUD insertions and most of the IUS insertions in the service take place at the city centre site (Central Abacus). In Abacus Clinics, the time taken for all consultations is recorded under three categories: less than 10 minutes, 10-20 minutes and more than 20 minutes.

We carried out an audit of the actual time taken for a complete IUD/IUS fitting service (instead of the usual three categories) at Central Abacus over a 4-week period in 2004 to see if the FFPRHC recommendations are an accurate reflection of what actually happens in our clinics. For the purposes of this audit, IUD/IUS provision in accordance with Good Medical Practice ${ }^{2}$ and Faculty

\section{Abacus Clinics for Contraception and Reproductive Health, Liverpool, UK \\ Varghese A George, MRCS, DFFP, Senior House Officer \\ Meera Kishen, MD, FFFP, Consultant in Family Planning and \\ Reproductive Health}

Correspondence to: Dr Meera Kishen, Central Abacus, Citrus House, 40-46 Dale Street, Liverpool L2 5SF, UK. Tel: +44 (0) 151284 2500. E-mail: meera.kishen@pct.northliverpool.nhs.uk
Standards included information provision to ensure informed decision-making, fitting procedure and related record keeping according to service protocol. All clinicians other than trainees who participated in the audit held the Faculty Letter of Competence in Intrauterine Techniques. The trainees were supervised by a Faculty Instructing Doctor during IUD/IUS fittings.

\section{Methods}

All clinicians carrying out IUD/IUS fittings in Central Abacus during the 4-week period from 1 June to 28 June 2004 were asked to complete a standard proforma relating to the time taken for different aspects of IUD/IUS provision. Clinicians were asked to record the actual time spent with the client at that visit and provide an allocation of time to each of three categories as follows:

- discussion/communication with the client

- insertion procedure including preparation of client/equipment and recovery

- record keeping.

The grade of the doctor as an indication of experience, any training activity in that consultation and any special clinical issues that might influence consultation time were also recorded. This information was analysed to ascertain the average total time taken for IUD/IUS provision, as well as the proportion of time taken for information provision, insertion and documentation elements of the service.

Three subgroups of the study population were identified: (1) women who reported an earlier visit to Abacus Clinics to discuss contraceptive choices or specifically IUD/IUS related to this insertion, (2) women who reported having used an IUD in the past and (3) women who had an IUD fitted as emergency contraception at the visit. Information relating to time taken for IUD/IUS service provision for these three groups was also analysed separately to ascertain whether there were any obvious differences in the time taken for each group as compared to the overall average time.

\section{Results}

A total of $61 \mathrm{IUD} / \mathrm{IUS}$ insertions were carried out at Central Abacus during the 4-week period from 1 June to 28 June 2004. A total of 41 completed proforma were received, giving a response rate of $67 \%$.

The average time taken for IUD/IUS provision was 35 minutes. Of this time, information provision took on average 12 minutes, insertion procedure 13 minutes and record keeping 10 minutes.

Fourteen of the 41 women reported an earlier visit to the service to discuss contraceptive choices or specifically the IUD/IUS. The remaining 27 women had the device fitted at their first visit requesting an intrauterine method. The effect of previous counselling on the average time taken for IUD/IUS provision is presented in Table 1. These figures do not include the time taken in the earlier visit related to this insertion.

Nine of the 41 women reported having used an IUD previously. Seven insertions were for emergency contraception. Twenty-five women had an IUD/IUS fitted for the first time with the intention of using it as their long- 
Table 1 Average time taken for intrauterine device/intrauterine system (IUD/IUS) provision: effect of previous counselling

\begin{tabular}{lll}
\hline Category & $n$ & Average time (min) \\
\hline Previous counselling visit & 14 & 33 \\
No previous counselling & 27 & 36 \\
Overall & 41 & 35 \\
\hline
\end{tabular}

Table 2 Average time taken for intrauterine device/intrauterine system (IUD/IUS) provision: effect of previous IUD use and indication for IUD

\begin{tabular}{lrl}
\hline Category & $n$ & Average time (min) \\
\hline Previous IUD use & 9 & 28 \\
First-time use as long-term method & 25 & 36 \\
Use as emergency contraception & 7 & 41 \\
Overall & 41 & 35 \\
\hline
\end{tabular}

Table 3 Average time taken for intrauterine device/intrauterine system IUD/IUS) provision: effect of training and grade of clinician

\begin{tabular}{lrl}
\hline Clinician grade & $n$ & Average time (min) \\
\hline Consultant/SCMO/AS & 17 & 30 \\
CMO/Staff Grade & 14 & 35 \\
Consultant/SCMO/AS with trainee & 3 & 40 \\
Trainee & 7 & 46 \\
Overall & 41 & 35
\end{tabular}

AS, associate specialist; CMO, clinical medical officer; SCMO, senior clinical medical officer.

term method of contraception. The effect of previous experience and indications for IUD use on the average time for IUD/IUS provision is presented in Table 2 .

The effect of grade of clinician and training activity on the average time taken for IUD/IUS provision is presented in Table 3. It should be noted that the senior clinicians in the service generally carry out all anticipated difficult insertions.

\section{Conclusions}

The average time taken for IUD/IUS provision at Central Abacus was $75 \%$ more than the minimum time recommended by the FFPRHC Service Standards. ${ }^{1}$ Training consultations required around twice as much time as the minimum recommendation. Emergency contraception IUD insertions took longer than insertions for long-term contraceptive use. Women who had previously used an intrauterine method took less time than those using an intrauterine method for the first time. Counselling for IUD use at an earlier visit did not appear to make much difference to the time taken in the subsequent consultation for IUD provision.

Those individuals responsible for setting standards and commissioners may wish to take these findings into account when deciding on future local and national services and recommendations.

\section{Statements on funding and competing interests Funding. None identified.}

Competing interests. None identified.

References

1 Faculty of Family Planning and Reproductive Health Care (FFPRHC) of the Royal College of Obstetricians and Gynaecologists. Service Standards for Workload in Contraception. London, UK, FFPRHC, May 2005. http://www.ffprhc.org.uk/admin/uploads/Service StandardsforWorkloadFINAL.pdf [Accessed 30 March 2006].

2 General Medical Council (GMC). Good Medical Practice. London, UK, GMC, May 2001 http:/www omc-uk org/guidance/good medical_practice/index.asp [Accessed 30 March 2006].

\section{termination of pregnancy}

\section{Respecting a woman's decision...}

\section{A leading charity in the field of reproductive healthcare, Marie Stopes International helps over one third of all women seeking termination of pregnancy services in the UK each year. We work closely with the NHS and also see private referrals.}

We are discreet, confidential and supportive and offer expert care and full information. Women who visit us will have a full consultation and assessment and a choice of options including same day consultation and treatment, choice of surgical or medical abortion and choice of anaesthetics.

Patients can rely on our $\mathbf{2 4}$ hour aftercare service and post abortion counselling service.

\section{Call us on 08451203644 - 24 hours}

for a GP information pack or visit

www.mariestopes.org.uk 